\title{
Thermal entanglement and efficiency of the quantum Otto cycle for the su(1,1) Tavis-Cummings system
}

\author{
L. Chotorlishvili ${ }^{1,3}$, Z. Toklikishvili ${ }^{2}$, J. Berakdar ${ }^{1}$ \\ 1 Institut für Physik, Martin-Luther Universität Halle-Wittenberg, Heinrich-Damerow-Str. 4 06120 Halle, Germany \\ 2 Physics Department of the Tbilisi State University, Chavchavadze av.3, 0128, Tbilisi, Georgia \\ 3 Institut für Theoretische Physik Universität Heidelberg Philosophenweg 19, D-69120 Heidelberg, Germany
}

\begin{abstract}
The influence of the dynamical Stark shift on the thermal entanglement and the efficiency of the quantum Otto cycle is studied for the su(1,1) Tavis-Cummings system. It is shown that the degree of the thermal entanglement becomes larger as the dynamical Stark shift increases. In contrast, the efficiency of the Otto cycle is degraded with an increase of the values of dynamical Stark shift. Expressions for the efficiency coefficient are derived. Using those expressions we identify the maximal efficiency of the quantum Otto cycle from the experimentally measured values of the dynamical Stark shift

PACS numbers:
\end{abstract}

\section{INTRODUCTION}

The Jaynes and Cummings (JC) 1] model is a paradigm of quantum optics describing a two-level system coupled to a single mode of the radiation field. Due to its relative simplicity and relevance to applications in diverse areas of modern optics such as cavity quantum electrodynamics [2, 3] and quantum state engineering with Josephson junction devices [4], JC model is still of current interest. The JC model and its generalizations [5-7] is particularly useful for studies in the field of quantum information processing 8]. A key issue in this context is the concept of the entanglement and the correlations between quantum states [9]. In recent years attention was devoted to so-called quantum engines 10 13 and the relation between the thermodynamics and the entanglement [14, 15]. In particular, two aspects are addressed: The relation between the degree of the entanglement and the efficiency of quantum engines [15] and the role of thermal fidelity as an indicator for a thermal phase transition [16, 17]. Generally, studies of thermodynamical effects in nanosized systems are expected to reveal the connection between quantum physics and thermodynamics contributing to the understanding of fundamental physical problems, such as the Maxwell's demon and the universality of the second law of thermodynamics [11]. Our focus here is on the so-called quantum heat engine that produces work based on quantum effects. For instance, for a quantum cavity one considers the quantum Otto cycle. This cycle contains two quantum adiabatic and two quantum isochoric parts and therefore preserves the volume of the cavity. As a theoretical model we utilize the su(1,1) Tavis-Cummings (TC) model [5] including the dynamical Stark shift (DSS) effect [6]. This model describes two-photon transitions between the ground and the excited state via an intermediate state. The intermediate state can be eliminated from the equations of motion [18, 19] on the cost of introducing a dynamical Stark shift [5]. Recently, the influence of DSS on the system's concurrence and fidelity was studied [20]. The main goal here is to study of the relation between the efficiency of the quantum Otto engine and DSS. We consider a quantum thermodynamical cycle for experimentally accessible situations. Specifically we consider a scenario where a system, consisting of atoms and a quantum cavity, is connected to two thermal baths one with a high and the other with a low temperature. As shown below, there is a direct relation between DSS and the efficiency of the quantum Otto engine; for a given DSS one can predict the efficiency of the quantum Otto engine and visa versa, i.e. from the evaluated efficiency one may infer the values of DSS.

\section{THERMAL CONCURRENCE}

The Hamiltonian of two TC atoms placed in an ideal cavity reads [5]

$$
\begin{aligned}
\hat{H} & =\left(\omega_{0}+\xi \hat{a}^{+} \hat{a}\right) S_{1}^{z}+\left(\omega_{0}+\xi \hat{a}^{+} \hat{a}\right) S_{2}^{z}+\omega \hat{a}^{+} \hat{a}+ \\
& +g\left[\left(S_{1}^{+}+S_{2}^{+}\right) \hat{a}^{2}+\left(S_{1}^{-}+S_{2}^{-}\right)\left(\hat{a}^{+}\right)^{2}\right]
\end{aligned}
$$

where $g$ is the coupling constant between the atoms and the radiation field of the cavity. $a$ and $a^{+}$are the photon annihilation and creation operators. $\xi$ is the strength of the Stark shift that results in an intensity dependent transition frequency. The atomic two-level systems are described by the spin operators $S^{z}=\frac{1}{2} \sigma_{z}$, $S^{ \pm}=\frac{1}{2}\left(\sigma_{x} \pm i \sigma_{y}\right)$, where $\sigma_{x, y, z}$ are Pauli operators. In (11) we neglect the kinetic energy $\frac{P^{2}}{2 M}$ of the center of mass of the atoms (with momentum $P$ and mass $M$ ). This is valid as long as $\frac{P^{2}}{2 M}<<d \sqrt{\frac{\hbar \omega n}{2 \varepsilon_{0} V}}$ [2], where $d$ is the atomic dipole moment, $V$ is the volume of the cavity, $\varepsilon_{0}$ is the electric constant and $n$ is the number of photons in the cavity. Due to the presence of the Stark shift term the eigenfrequency of the system is not constant anymore. Usually similar problems arise in non-stationary dynamics of nonlinear systems, where the frequency shift has 
a crucial consequences [21]. Here in a stationary problem we also expect to see consequences of the frequency shift. As the Stark shift term changes the system's energy spectrum it should also affect the thermodynamic characteristics of the quantum cycle. In the limit of strong $g \gg \zeta$ or weak $g \ll \zeta$ coupling regimes between radiation field of cavity and atomic subsystem, in the interaction representation Hamiltonian (1) splits in two commuting parts [20]. However, for moderate $\zeta \sim g$ we expect to see strong correlation effects. As usual, the radiation filed is assumed to be prepared in a coherent state with the distribution function $W_{n}^{2}=\frac{\alpha^{2 n}}{n !} e^{-\alpha^{2}}$, where $\alpha$ is the mean photon number $n$. We use the following notation for the relevant Hilbert space vectors ( $g$ and $e$ stand for the ground and excited state)

$$
|e g, n+2\rangle, \quad|g e, n+2\rangle, \quad|e e, n\rangle, \quad|g g, n+4\rangle .
$$

In this basis and for the resonant case, i.e. for $\omega_{0}=2 \omega$, we construct density operator of the system. Since working substance of the Otto engine is formed by two TC atoms we are interested in the density operator of the atomic subsystem. Note that the field is the subsystem with a large number of degrees of freedom and is prepared in a coherent state. Therefore, the state of the field is not influenced by the atom-field coupling interaction. In particular the state of the field can never be identified exactly due to its probabilistic nature. Consequently, for quantifying the entanglement between the atoms we should average and trace out the field states which leads to a loss of coherence between the atomic and the field subsystems. Nevertheless, the atomic states are still correlated and we expect to obtain a nonzero concurrence. In the language of density matrix, the coherence of the atomic states means non-vanishing off-diagonal matrix elements of the reduced density matrix. In addition to the connection between the engine efficiency and the thermal concurrence, further important issues to be discussed are the consequences of the system's transition form pure to the mixed state on the engine efficiency. Using basis vectors (2) after tracing field states for the reduced density operator of the atomic subsystem $\rho_{a}=\operatorname{Tr}_{f}\left(\frac{e^{-\beta H_{i n t}}}{Z}\right)$ we deduce

$$
\begin{aligned}
\rho_{11}= & \frac{1}{Z} \frac{2 b^{2}}{\left(a^{2}+4 b^{2}\right)}+\frac{1}{Z} \frac{\left(a^{2}+2 b^{2}\right) \cosh \left[\beta \sqrt{a^{2}+4 b^{2}}\right]}{a^{2}+4 b^{2}}- \\
\frac{1}{Z} & \frac{a \sinh \left[\beta \sqrt{a^{2}+4 b^{2}}\right]}{\sqrt{a^{2}+4 b^{2}}}, \\
\rho_{22}= & \frac{1}{Z} \frac{2 b^{2}}{\left(a^{2}+4 b^{2}\right)}+\frac{1}{Z} \frac{\left(a^{2}+2 b^{2}\right) \cosh \left[\beta \sqrt{a^{2}+4 b^{2}}\right]}{a^{2}+4 b^{2}}+ \\
\frac{1}{Z} & \frac{a \sinh \left[\beta \sqrt{a^{2}+4 b^{2}}\right]}{\sqrt{a^{2}+4 b^{2}}}, \\
\rho_{12}= & \rho_{21}=\rho_{34}=\rho_{43}= \\
\frac{1}{Z} & \frac{2 b^{2}\left(-1+\cosh \left[\beta \sqrt{a^{2}+4 b^{2}}\right]\right)}{a^{2}+4 b^{2}},
\end{aligned}
$$

$$
\begin{aligned}
\rho_{13}= & \rho_{14}=\rho_{31}=\rho_{41}= \\
& \frac{1}{Z} \frac{a b\left(-1+\cosh \left[\beta \sqrt{a^{2}+4 b^{2}}\right]\right)}{a^{2}+4 b^{2}} \\
- & \frac{1}{Z} \frac{b \sinh \left(\beta \sqrt{a^{2}+4 b^{2}}\right)}{\sqrt{a^{2}+4 b^{2}}}, \\
\rho_{23}= & \rho_{32}=\rho_{24}=\rho_{42}=\frac{1}{Z} \frac{a b\left(1-\cosh \left[\beta \sqrt{a^{2}+4 b^{2}}\right]\right)}{a^{2}+4 b^{2}}- \\
\frac{1}{Z} & b \sinh \left(\beta \sqrt{a^{2}+4 b^{2}}\right) \\
\rho_{33}= & \rho_{44}=\frac{1}{Z} \frac{a^{2}+b^{2}+2 b^{2} \cosh \left[\beta \sqrt{a^{2}+4 b^{2}}\right]}{a^{2}+4 b^{2}} .
\end{aligned}
$$

The partition function reads

$$
Z=\operatorname{Tr}\left(e^{-\beta H}\right)=2\left(1+\cosh \left(\beta \sqrt{a^{2}+4 b^{2}}\right)\right) .
$$

Here $a=\xi \alpha^{2}, b=g \alpha^{2}$. The eigenvalues of the Hamiltonian (11) are

$$
E_{1}=-\sqrt{a^{2}+4 b^{2}}, E_{2}=0, E_{3}=0, E_{4}=\sqrt{a^{2}+4 b^{2}} .
$$

Using (3),(4) we follow Ref. 22] and evaluate the concurrence $C$ according to

$$
C=\max \left(0, \sqrt{R_{1}}-\sqrt{R_{2}}-\sqrt{R_{3}}-\sqrt{R_{4}}\right)
$$

where the square roots correspond to the eigenvalues of the matrix

$$
R=\left(\sigma_{y} \otimes \sigma_{y}\right) \rho^{*}\left(\sigma_{y} \otimes \sigma_{y}\right) \rho .
$$

As we seek to inspect the influence of the Stark shift we consider an asymptotic case that corresponds to large values of the Stark shift $g<<\xi$. In this case eqs. (3) and (4) simplify and we obtain

$$
\begin{gathered}
\rho=\frac{1}{Z}\left(\begin{array}{cccc}
e^{-\beta a} & 0 & 0 & 0 \\
0 & e^{\beta a} & 0 & 0 \\
0 & 0 & 1 & 0 \\
0 & 0 & 0 & 1
\end{array}\right) \\
R=\frac{1}{Z^{2}}\left(\begin{array}{cccc}
e^{-\beta a} & 0 & 0 & 0 \\
0 & e^{\beta a} & 0 & 0 \\
0 & 0 & e^{\beta a} & 0 \\
0 & 0 & 0 & e^{-\beta a}
\end{array}\right), \\
Z=2(1+\cosh \beta a) .
\end{gathered}
$$

Furthermore, we obtain for the eigenvalues

$$
R_{1,2}=\frac{e^{\beta a}}{Z^{2}}, R_{3,4}=\frac{e^{-\beta a}}{Z^{2}}, R_{1,2}>R_{3,4}
$$


and therefore

$$
C=\max \left(0,-2 \sqrt{R_{3}}\right)=0 .
$$

Obviously a large Stark shift leads to a disentanglement. The same result we obtain in the case of a small Stark shift. Indeed, considering $g>>\xi$ we infer from eq. (3) and (4) that

$$
\rho=\left(\begin{array}{cccc}
\frac{1}{4} & a_{1} & a_{2} & a_{2} \\
a_{1} & \frac{1}{4} & a_{2} & a_{2} \\
a_{2} & a_{2} & \frac{1}{4} & a_{1} \\
a_{2} & a_{2} & a_{1} & \frac{1}{4}
\end{array}\right)
$$

In what follows we introduce the following notations

$$
a_{1}=\frac{1}{2 Z}(-1+\cosh 2 \beta b), a_{2}=-\frac{1}{2 Z} \sinh 2 b \beta,
$$

and

$$
Z=2(1+\cosh 2 b \beta) .
$$

The matrix $R$ and its eigenvalues read

$$
R=\left(\begin{array}{llllr}
\frac{1}{16}-a_{1}^{2} & 0 & 0 & \frac{a_{2}}{2}-2 a_{1} a_{2} \\
0 & \frac{1}{16}-a_{1}^{2} & \frac{a_{2}}{2}-2 a_{1} a_{2} & 0 \\
0 & \frac{a_{2}}{2}-2 a_{1} a_{2} & \frac{1}{16}-a_{1}^{2} & 0 \\
\frac{a_{2}}{2}-2 a_{1} a_{2} & 0 & 0 & \frac{1}{16}-a_{1}^{2}
\end{array}\right)
$$

$$
R_{1,2}=\frac{e^{6 \beta b}}{\left(1+e^{2 \beta b}\right)^{2}}, R_{3,4}=\frac{e^{2 \beta b}}{\left(1+e^{2 \beta b}\right)^{2}}, \text { and hence }
$$

$$
C=\max \left(0,-2 \sqrt{R_{3}}\right)=0
$$

This Result is quite simple and evident. The value of DSS is the key factor since for both limiting cases the system is disentangled. To consider arbitrary values of DSS we introduce the following notations

$$
\begin{aligned}
x & =\frac{2 b}{a}=\frac{2 g}{\xi}, \quad y=\beta a=\frac{\xi \alpha^{2}}{T} \\
b_{1} & =\frac{1}{2} \frac{x \cosh \left[y \sqrt{1+x^{2}}\right]}{1+x^{2}} \\
b_{2} & =\frac{1}{2} \frac{x \sinh \left[y \sqrt{1+x^{2}}\right]}{1+x^{2}} .
\end{aligned}
$$

and rewrite the density matrix in the form

$$
\begin{gathered}
\rho=\frac{1}{Z}\left(\begin{array}{cccc}
1+x b_{1}+\frac{2}{x}\left(b_{1}-b_{2}\right) & x b_{1} & \left(b_{1}-b_{2}\right) & \left(b_{1}-b_{2}\right) \\
x b_{1} & 1+x b_{1}+\frac{2}{x}\left(b_{1}+b_{2}\right) & -\left(b_{1}+b_{2}\right) & -\left(b_{1}+b_{2}\right) \\
\left(b_{1}-b_{2}\right) & -\left(b_{1}+b_{2}\right) & \left(1+x b_{1}\right) & x b_{1} \\
\left(b_{1}-b_{2}\right) & -\left(b_{1}+b_{2}\right) & x b_{1} & 1+x b_{1}
\end{array}\right) \\
Z=2\left(1+\cosh y \sqrt{1+x^{2}}\right)
\end{gathered}
$$

Consequently, the matrix $R=\left(\sigma_{y} \otimes \sigma_{y}\right) \rho^{*}\left(\sigma_{y} \otimes \sigma_{y}\right) \rho$ reads

$$
R=\frac{1}{Z^{2}}\left(\begin{array}{cccc}
\frac{\left(1+2 b_{1} x\right)\left(x+2\left(b_{1}-b_{2}\right)\right)}{x} & 4 b_{1}\left(-b_{1}+b_{2}\right) & \frac{2 b_{1}\left(2 b_{1}+x\right)-4 b_{2}^{2}}{x} & \frac{2\left(x+2\left(b_{1}-b_{2}\right)\right)\left(b_{1}-b_{2}\right)}{x} \\
-4 b_{1}\left(b_{1}+b_{2}\right) & \frac{\left(1+2 b_{1} x\right)\left(x+2\left(b_{1}+b_{2}\right)\right)}{x} & \frac{-2\left(b_{1}+b_{2}\right)\left(x+2\left(b_{1}+b_{2}\right)\right)}{x} & \frac{-2 b_{1}\left(2 b_{1}+x\right)+4 b_{2}^{2}}{x} \\
2 b_{1}\left(1+2 b_{1} x\right) & -2\left(4 b_{1}^{2} x+\left(b_{1}+b_{2}\right)\right) & \frac{\left(1+2 b_{1} x\right)\left(x+2\left(b_{1}+b_{2}\right)\right)}{x} & 4 b_{1}\left(b_{1}-b_{2}\right) \\
2\left(b_{1}-b_{2}+2 b_{1}^{2} x\right) & -2 b_{1}\left(1+2 b_{1} x\right) & 4 b_{1}\left(b_{1}+b_{2}\right) & \frac{\left(1+2 b_{1} x\right)\left(x+2\left(b_{1}-b_{2}\right)\right)}{x}
\end{array}\right) .
$$

The eigenvalues of the Matrix (12) and consequently the thermal concurrence is a function of two parameters: The first parameter is the relation between the cavityatom coupling constant and the Stark shift $x=\frac{2 g}{\xi}$. The second one describes the temperature dependence of the concurrence $y=\frac{\xi \alpha^{2}}{T}$. Fig.1 is deduced from the calculations using (13). From this figure we conclude that the dependence on the first parameter is more relevant for the thermal concurrence. Therefore, the relation be- tween the cavity-atom coupling constant and the Stark shift is the key parameter. In the limiting cases $\xi>>g$, and $\xi<<g$, as was shown before analytically, the thermal concurrence is zero. The concurrence increases linearly with the parameter $g / \xi$ up to the maximal value $C_{\max }(g / \xi) \approx 0.5$. This fact can be explained analytically. Considering in eqs. (3) and (4) $g^{2} / \xi^{2}$ as a small parameter and $a \beta$ as a large parameter and retaining only the first order terms we find that $R$ matrix reduces to 


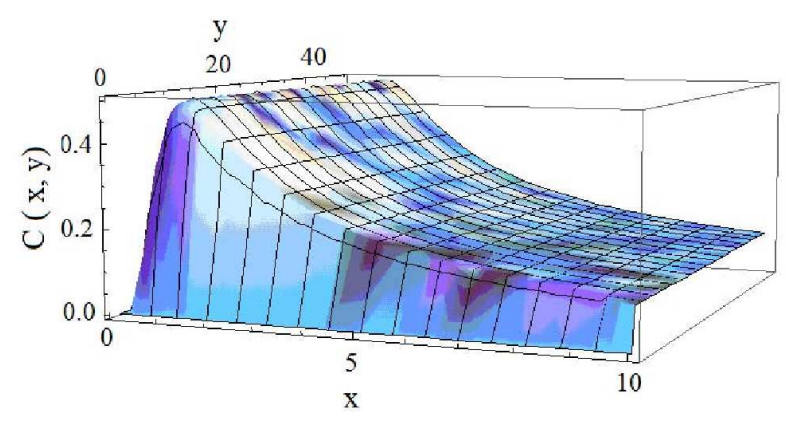

FIG. 1: Thermal concurrence as a function of the parameter $x=\frac{2 g}{\xi}, y=\frac{\xi \alpha^{2}}{T}$. For large and small of Stark shifts the system is not entangled. A maximal concurrence value is achieved for an intermediate Stark shift.

$$
R=\left(\begin{array}{cccc}
0 & 0 & 0 & 0 \\
-2 g^{2} / \xi^{2} & 2 g^{2} / \xi^{2} & 2 g / \xi & 0 \\
0 & 0 & g^{2} / \xi^{2} & 0 \\
0 & 0 & 2 g^{2} / \xi^{2} & 0
\end{array}\right)
$$

Consequently the expression for the concurrence $C(g / \xi)=\max (0, g / \xi(\sqrt{2}-1))$ is in line with the numerical result. In the opposite case, i.e. for $\xi<g$, and $\frac{b \beta}{(g / \xi)^{2}} \geqslant 1$ we infer for the concurrence a square root decay

$$
\begin{aligned}
C(g / \xi)= & \max \left(0, \sqrt{\frac{2 b \beta}{(g / \xi)^{2}}-1+\sqrt{\frac{2 b \beta}{(g / \xi)^{2}}-1}}\right. \\
& -\sqrt{\frac{2 b \beta}{(g / \xi)^{2}}-1-\sqrt{\left.\frac{2 b \beta}{(g / \xi)^{2}}-1\right)}},
\end{aligned}
$$

which is also in a good agreement with the numerical results. Namely in the regime of weak coupling between atom and cavity concurrence is linear function of $g / \xi$. In the regime of strong coupling we have square root law for decay of concurrence.

\section{EFFICIENCY OF QUANTUM OTTO ENGINE AND THE DYNAMICAL STARK SHIFT}

Having defined the thermal concurrences for our system we focus now on the relation between the values of the Stark shift, the concurrence and the efficiency of the quantum Otto engine. A quantum Otto engine with two two-level atoms as a working substance was proposed and discussed in details in [15]. Here we recall very briefly the main ideas: Two TC atoms are placed in an ideal lossless cavity which is connected to two thermal baths with different temperatures $\beta_{L}=\frac{1}{T_{L}}, \beta_{H}=\frac{1}{T_{H}}$. The system performs a quantum Otto cycle consisting of two adiabatic and two isochoric parts. The heat exchange with

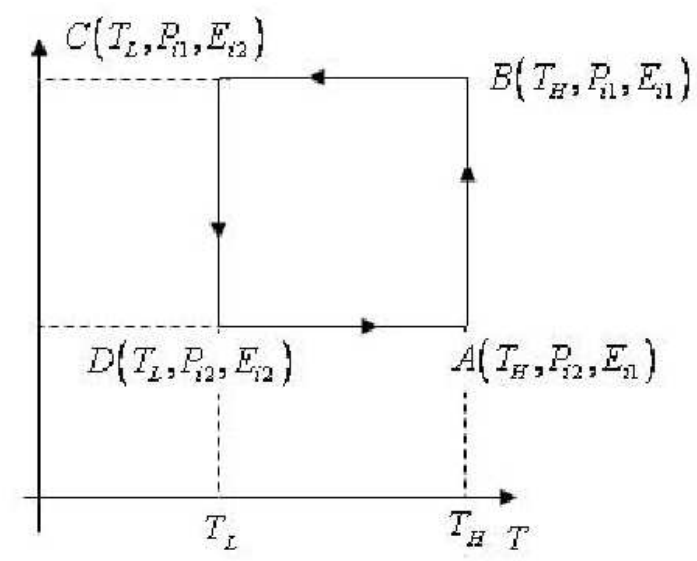

FIG. 2: 1) $A \rightarrow B$ system is connected with hot reservoir $T_{H}$, structure of energy levels is conserved $E_{i 1}$ while populations are changed from $P_{i 2} \quad(i=1,2,3,4)$ to $P_{i 1} \quad(i=1,2,3,4)$ Work performed is zero. Isochoric part

2) $B \rightarrow C$ Adiabatic part, level populations is conserved $P_{i 1} \quad(i=1,2,3,4)$. While energy levels are changed from $E_{i 1} \quad(i=1,2,3,4)$-to $E_{i 2}(i=1,2,3,4)$

3) $C \rightarrow D$ is the reverse process of $A \rightarrow B$

4) $D \rightarrow A$ adiabatic part. Populations are conserved $P_{i 2}(i=$ $1,2,3,4)$ energy levels changed $E_{i 2}(i=1,2,3,4)$-from to $E_{i 1}(i=1,2,3,4)$

the thermal reservoir changes the population of the levels $P_{i j} \quad(i=1,2,3,4 . j=1,2)$ (isochoric part), while during the adiabatic parts the performed work changes the structure of the energy terms $E_{i j} \quad(i=1,2,3,4 . j=1,2)$ (due to the change of the atom-cavity coupling constant [23]). For $P_{i j}$ the first index $i$ enumerates the level population probabilities, while the second index denotes the probabilities before or after the heat transfer from the thermostat to the system. For $E_{i j}$ the first index describes the energy levels while the second defines the energy spectrum before or after performing work (i.e., the performed work changes the structure of the energy spectrum as was stated above.) The quantum Otto cycle is shown schematically in Fig.2. Following the standard notations, e.g. as in [15], we write

$$
\begin{gathered}
E_{i 1}=\left\{\begin{array}{l}
E_{11}=-\lambda_{1} ; \\
E_{21}=0 ; \\
E_{31}=0 ; \\
E_{41}=\lambda_{1} ;
\end{array} \quad E_{i 2}=\left\{\begin{array}{l}
E_{11}=-\lambda_{2} ; \\
E_{21}=0 ; \\
E_{31}=0 ; \\
E_{41}=\lambda_{2} ;
\end{array}\right.\right. \\
P_{i 1}=\left\{\begin{array}{l}
P_{11}=\exp \left(+\beta_{H} \lambda_{1}\right) / Z_{H} ; \\
P_{21}=\frac{1}{Z_{H}} \\
P_{31}=\frac{1}{Z_{H}} \\
P_{41}=\exp \left(-\beta_{H} \lambda_{1}\right) / Z_{H} ;
\end{array}\right.
\end{gathered}
$$




$$
P_{i 2}=\left\{\begin{array}{l}
P_{12}=\exp \left(+\beta_{L} \lambda_{2}\right) / Z_{H} \\
P_{22}=\frac{1}{Z_{L}} \\
P_{32}=\frac{1}{Z_{L}} \\
P_{41}=\exp \left(-\beta_{L} \lambda_{2}\right) / Z_{L}
\end{array}\right.
$$

Here

$$
\begin{gathered}
\lambda_{1}=\alpha^{2} \sqrt{\xi^{2}+4 g_{1}^{2}}, \lambda_{2}=\alpha^{2} \sqrt{\xi^{2}+4 g_{2}^{2}}, a=\alpha^{2} \xi, \\
Z_{H}=2\left(1+\cosh \beta_{H} \lambda_{1}\right),
\end{gathered}
$$

and

$$
Z_{L}=2\left(1+\cosh \beta_{H} \lambda_{2}\right) .
$$

Using (14),(15) and the standard definitions of the thermodynamic quantities such as the energy of the system $E$, and the transferred heat $Q$ as well as the work performed $A$ we find

$$
\begin{aligned}
& E=\sum_{i=1}^{4} E_{i j} P_{i j} \\
& d E=\sum_{i=1}^{4} E_{i j} d P_{i j}+\sum_{i=1}^{4} P_{i j} d E_{i j}, \\
& d Q=\sum_{i=1}^{4} E_{i j} d P_{i j}, \\
& d A=\sum_{i=1}^{4} P_{i j} d E_{i j}, \\
& Q_{H}=Q_{A B}=\int_{A}^{B} \sum_{i=1}^{4} E_{i 1} d P_{i j} \\
& =\sum_{i=1}^{4} E_{i 1}\left(P_{i 1}-P_{i 2}\right)= \\
& =2 \lambda_{1}\left(\frac{\sinh \beta_{L} \lambda_{2}}{Z_{L}}-\frac{\sinh \beta_{H} \lambda_{1}}{Z_{H}}\right) \\
& Q_{L}=-Q_{C D}=-\int_{C} \sum_{i=1}^{4} E_{i 2} d P_{i j} \\
& =-\sum_{i=1}^{4} E_{i 2}\left(P_{i 2}-P_{i 1}\right)= \\
& =2 \lambda_{2}\left(\frac{\sinh \beta_{H} \lambda_{1}}{Z_{H}}-\frac{\sinh \beta_{L} \lambda_{2}}{Z_{L}}\right)=\frac{\lambda_{2} Q_{H}}{\lambda_{1}}
\end{aligned}
$$

Finally for the efficiency coefficient we obtain

$$
\begin{aligned}
& \eta=\frac{Q_{H}-Q_{L}}{Q_{L}}=1-\frac{\lambda_{2}}{\lambda_{1}}=1-\frac{\sqrt{1+\left(g_{2} / \xi\right)^{2}}}{\sqrt{1+\left(g_{1} / \xi\right)^{2}}}(20) \\
& g_{1}=g+\delta g, g_{2}=g .
\end{aligned}
$$

For the small values of the Stark shift we arrive at the conclusions

$$
\eta(\xi<<g)=\frac{Q_{H}-Q_{L}}{Q_{H}}=\frac{\delta g}{g}+\left(\frac{\delta g}{g}\right)^{2}+\ldots,
$$

while for large shift we deduce

$$
\eta(\xi>>g)=\frac{Q_{H}-Q_{L}}{Q_{H}} \approx \frac{(\delta g) g}{\xi^{2}},
$$

$$
\eta(\xi>>g)<\eta(\xi<<g) .
$$

Efficiency of Otto engine $\eta(\xi),(20)$ has a maximum for zero values of the Stark shift

$$
\eta_{\max }=\eta(\xi=0)=1-g /(g+\delta g),
$$

when thermal concurrence is zero. Therefore we can argue that quantum correlations in the atomic subsystem hinders realization of quantum Otto cycle with maximal efficiency.

\section{CONCLUSIONS}

The purpose of the present paper has been to study the influence of DSS on the thermal entanglement and the efficiency of the quantum Otto cycle. For this purpose we considered the $\mathrm{su}(1,1)$ Tavis-Cummings system and showed that the degree of the thermal entanglement follows the values of the DSS. In particular, for vanishing DSS, i.e. $\xi=0$ the system is disentangled, and the degree of the thermal entanglement increases with DSS. The system becomes disentangled again only in the limit $\xi \gg g$, i.e. for a weak atom-cavity coupling. The efficiency of the quantum Otto cycle is maximal for a small values of $\operatorname{DSS} \eta(\xi<<g)>\eta(\xi>>g)$, i.e. when system is disentangled. On the other hand, $\eta$ decreases as $\eta(\xi) \approx 1 / \xi^{2}$. Using the asymptotic expressions for the efficiency coefficient (20), one may identify the maximal efficiently of the quantum Otto cycle from the experimentally measured DSS. For $\xi<<g$, the efficiency is $\eta \sim \delta g / g$, where $\delta g$ is the amendment of the atom cavity coupling constant due to the performed work. If $\xi>>g$ then we obtain for the efficiency $\eta \sim(\delta g) g / \xi^{2}$. For a given value of the DSS, one can quantify the thermal concurrence as well. Again the key point is the relation $\xi / g$. For $\xi / g<1$, the concurrence is given in an explicit analytical expression $C(g / \xi)$ see Eq. (14). For the opposite case $\xi / g>1$ we refer to the expression above. In the strong coupling limit $\xi<<g$ or large DSS $\xi>>g$ system is disentangled. From the physical point of view this result is expectable. In both cases the Hamiltonian of the system (1) can be presented as the sum of two commuting parts $\hat{H}_{\text {int }}=\hat{H}_{1}\left(s_{1}^{z}, s_{1}^{ \pm}\right)+\hat{H}_{2}\left(s_{2}^{z}, s_{2}^{ \pm}\right),\left[\hat{H}_{1}, \hat{H}_{2}\right]=0$. Therefore, the reduced density matrix of the atomic subsystem is a separable $\rho_{a}=\rho_{1} \oplus \rho_{2}$. In the language of thermal entanglement both limits are identical (entanglement is zero), while the engine works with the maximal efficiency only for $\xi<<g$. Therefore, we can conclude that a zero entanglement is essential but not sufficient a criteria to reach the maximal efficiency. Comparing Eq. (3) with the Eq. (5) we see that with the increase of 
DSS the system performs a transition from a pure state to a mixed state where the non diagonal elements of the density matrix are zero Eq.(5). The system produces maximal efficiency for $\xi<<g$, being in the pure state Eq. (8). Therefore, we can conclude that for a maximal efficiency the working substance should be in a pure coherent state and the transition to the mixed state degrades the efficiency of the quantum engine.

Acknowledgments The financial support by the Deutsche Forschungsgemeinschaft (DFG) through SFB 762, the Grant No. KO-2235/3 and STCU Grant No. 5053 is gratefully acknowledged.
[1] E. Jaynes, F. Cummings, Proc. IEEE 51, 89 (1963).

[2] P. Schleich, Quantum Optics in Phase Space, WileyVCH, Berlin, 2001.

[3] J. Raimond, M. Brune, S. Haroche, Rev. Mod. Phys. 73, $565(2001)$

[4] Y. Makhlin, G. Schön, A. Shnirman, Rev. Mod. Phys. 73, 357 (2001).

[5] A. Joshi, R.R. Puri, J. Mod. Opt. 36, 215 (1989), H. Moya-Cessa, P. L. Knight, and A. Rosenhouse-Dantsker Phys. Rev. A 50, 1814 (1994).

[6] D. Zueco, G. M. Reuther, S. Kohler, and P. Hänggi Phys. Rev. A 80, 033846 (2009).

[7] A. Rybin, G. Kastelewicz, J. Timonen, N. Bogoliubov, J. Phys. A Math. Gen. 31, 4705. (1998).

[8] H. Mabuchi, A. Doherty, Science 298, 1372 (2002).

[9] L. Amico, R. Fazio, A. Osterloh, V. Vedral, Rev. Mod. Phys. 80, 517 (2008).

[10] S.N. Shevchenko, S. Ashhab, F. Nori, Phys. Reports 492, 1 (2010).

[11] K. Maruyama, F. Nori, V. Vedral, Rev. Mod. Phys. 81, 1 (2009).

[12] I. Buluta, F. Nori, Science 326, 108 (2009).
[13] P. Hänggi, F. Marchesoni, F. Nori Annalen der Physik 14, 51 (2005).

[14] V. Vedral and E. Kashefi, Phys. Rev. Lett. 89, 037903 (2002).

[15] H. T. Quan, Y. X. Liu, C. P. Sun and F. Nori, Phys. Rev. E 76, 031105 (2007).

[16] H. T. Quan and F. M. Cucchietti, Phys. Rev. E 79, 031101 (2009).

[17] P. Zanardi, H. T. Quan, X. Wang, and C. P. Sun Phys. Rev. A 75, 032109 (2007).

[18] P. Alsing, M.S. Zubairy, J. Opt. Soc. Am. B 4, 177 (1987).

[19] R.R. Puri, R.K. Bullough, J. Opt. Soc. Am. B 5, 2021 (1988).

[20] L. Chotorlishvili, P. Schwab, Z. Toklikishvili V. Skrinnikov, Phys. Lett. A 374, 1642, (2010).

[21] L. Chotorlishvili P. Schwab, Z. Toklikishvili, J. Berakdar J. Comp. Th. Nanoscience 7, 2430-2440 (2010).

[22] W. K. Wootters, Phys. Rev. Lett. 80, 2245 (1998).

[23] H. Wang, S. Liu, and J. He, Phys. Rev. E 79, 041113 (2009). 